\title{
The Role of Cardiac MRI in the Postsurgical Follow Up of Aortic Coarctation-Our Experience
}

\author{
Stuppner S* and Stuefer J \\ Department of Diagnostic and Interventional Radiology, General Hospital Bolzano, \\ Italy
}

*Corresponding author: Sigmund Stuppner, Department of Diagnostic and Interventional Radiology, General Hospital Bolzano, Lorenz Boehler Street, 5-39100 Bolzano, Italy, Tel: 0039/0471/908494; Email: stuppnersigmund@web.de

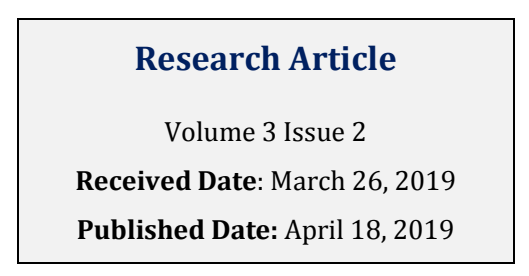

0039/0471/908494; Email: stuppnersigmund@web.de

\section{Abstract}

Congenital cardiac disease represents the most frequent malformation pathology, with an incidence variable from $0.5 \%$ to $1.2 \%$ of live births. These values are significantly higher if we also consider mild congenital defects that do not require treatment or are not recognized at birth, but which can become clinically relevant in adulthood, such as some abnormalities of the aortic arch, the bicuspid aortic valve, an abnormal venous return partial, coronary anomalies and fistulas. In our South Tyrol region (Italy), the birth rate is of about 5500 births / year, equal to a prevalence of CCD of 50 60 / year. About $40 \%$ of the CCDs requires surgical treatment in the neonatal period ( $<30$ days) and about $90 \%$ of patients operated reach adulthood. The rate of growth of the adult population affected by CCD is equal to $5 \%$ per annum. The purpose of this study is to present our experience in the morphological and MRI study functional in the postoperative follow-up of aortic coarctation.

Keywords: Cardiac MRI; Aortic Coarctation; CMR examination

Abbreviations: CCD: Congenital Cardiac Disease; HASTE: Half Fourier Acquisition Single shot Turbo Spin Echo; SSFSE: Single Shot Fast Spin Echo; CMR: Cardiac Magnetic Resonance; AC: Aortic Coarctation; TT: Termino Terminal Anastomosis; AI: Aortic Insufficiency; ARF: Aortic Regurgitation Fraction; SP: Status Post; TEE: Transesophageal Echocardiography; TR: Time Repetition Time; TE: Echo Times; SPGR: Spoiled Gradient Echo.

\section{Introduction}

In the follow-up of adult patients with CoA after surgery palliative and /or corrective the cardiac magnetic resonance (CMR- cardiovascular magnetic resonance) reached a vast potential after at least thirty years of evolution. The improvement of the software and hardware of the topographers, and the development of strong gradients, phased array coils and fast sequences after the 1990s, today allow an accurate non-invasive diagnosis of morphology, function, perfusion and vitality of the cardiovascular system. In competition with transthoracic and transesophageal echocardiography (TEE), with conventional angiography and cardiopulmonary scintigraphy, CMR has realized a cardiologist's dream: without the use of ionizing radiation high-resolution images of the heart are obtained in movement, of the valves, of the large mediastinal and pulmonary vessels and of the coronary arteries. In addition to morphological information, CMR offers a 


\section{Clinical Radiology \& Imaging Journal}

series of functional information: The Cine sequences allow a quantification of the systolic and diastolic function global and regional. Phase-Contrast sequences allow a qualitative and quantitative study in any vascular district. They give us information about flow velocity, flow volumes and on transvalvular flowmetric profiles and therefore allow a quantification of regurgitations evalvular stenosis, vascular stenosis, intra- and extracardiac shunts. Allow in competition for scintigraphy is a quantification of lung perfusion $[1,2]$.

CMR is like echocardiography a non-invasive technique, but it offers more than diagnostics cardiology the possibility of the study of extracardiac, mediastinal and vascular structures pulmonary and with the technological improvement of hardware and software has become an exam of "Gold standard" in the study of congenital heart disease (CCD) operated. Important advances in palliative and corrective medical and surgical treatment and in the CCD interventional procedures in recent decades have made expectations higher life of these patients with an increase in the prevalence of operated and congenital heart disease of the number of those who need continuous monitoring both to recognize early post-surgical complications and residual defects both to identify the possible recurrence of a malformation aspect (for example the re-classification).

\section{Material and Methods}

The CMR examination was performed on a $1.5 \mathrm{~T}$ device (Avanto, Siemens) equipped with a system of highspeed ascent gradients $(150 \mathrm{~m} / \mathrm{T} / \mathrm{sec}$ with a peak of 30 $\mathrm{T} / \mathrm{m}$ ). The exam requires a multi-channel phase-array surface coil, of which we have an arrangement of a 5element coil. It is primarily used for fast and fast imaging increase the signal-to-noise ratio (S/R) with parallel imaging with the SENSE (Sensitive Encoding Technique) approach [3]. The processing of the functional data and of the angiographic data took place with a special software Syngo - MR from Siemens, the cardiovascular functionality data in ARGUS software and Angio data-3D MR with vascular 3D software. The examination is performed with the patient supine and the electrodes for cardio synchronization are positioned appropriately on the anterior chest wall. To obtain morphological, dynamic and functional diagnostic images of the heart, of vascular structures, data acquisition must be adapted to physiological conditions of the intrinsic movement of the heart during the cardiac cycle and of the movement of the structures cardiovascular during breath. For the study of the heart they are therefore indispensable besides ultrafast sequences, also cardio synchronization systems using the electrocardiogram and synchronization systems of the acquisition with respiratory movement (navigator respiratory). The cardio synchronized acquisition allows the acquisition of data in a defined interval of the cardiac cycle away from the $\mathrm{R}$ wave and allows synchronizing the acquisition to the movements of the heart and the pulsatile movement of the blood flow. It therefore allows not only for obtain static images always in the same phase of the cardiac cycle, but also images of the moving heart (CINE images).

\section{Cardio-Dedicated Sequences}

Eco Spin Sequences (SE) with black blood images (db "dark blood"): The SE type sequences are now acquired with the Turbo Spin Eco technique (TSE = Fast Spin Eco FSE) or single shot (HASTE or SSFSE). The introduction of the TSE technique allowed for the first time to obtain images with weighing in T2. They are acquired in apnea and single layer, while with the HASTE technique they can be obtain multiple layers in a single apnea phase at the expense of contrast resolution. The "dark blood" technique allows to reduce flow artifacts and cardiac movements obtaining a lumen of the vessels and cardiac cavities of black color. Acquisitions must be suitable for heart rate in such a way that they are acquired at the end of the diastole phase of the cycle heart.

GRE sequences with "white blood" images [4,5]: In GE sequences the signal echo is produced by gradients and not by the radiofrequency pulse. In GE sequences used in CMR the TR is very short and less than the blood T1 (long T2 and short T1) which explains the high signal (white) of the blood ("white blood"), regardless of the flow effect. This determines an optimal contrast between myocardium and the circulating blood, and allows times of extremely limited acquisitions. In CMR we essentially use two types of fast GE sequences (FGE = fast GE) with retrospective cardio synchronization:

a) Flash ("fast low angle shot"), also called "spoiled" - GRE (gradient echo) refocused), in which the residual transverse magnetization is not refocused, but eliminated ("spoiled").

b) True Fisp ("Fast Imaging in the Steady State Precession"), also called SSFP (Steady state free precession) coherent, in which the residual transverse magnetization is refocused to contribute to the equilibrium state between longitudinal and transverse magnetization. 


\section{Clinical Radiology \& Imaging Journal}

\section{Study Mode}

The cardiovascular study in the follow-up of CoA has the aim to investigate different objectives with different types of sequences:

a) Anatomical orientation study

b) Anatomical study of the heart

c) Anatomical study of large vessels

d) Study of the biventricular function

e) Quantitative flow study

\section{Orientative Anatomical Study}

Each CMR exam begins with the study of the anatomy and morphology of mediastinal structures and thoracic in the three orthogonal planes of the body (axial, coronal and sagittal). In this regard we use sequences with multiple layer measurements, which allow acquisition of a packet of layers in a plane (about 12-15 slices) in a single apnea phase lasting 15-22 seconds. They are acquired with the "dark blood" technique weighed in T1 (TSE T1) or T2 (HASTE) or "white blood" SSFP sequences.

\section{Anatomical Study of the Heart}

The heart is not oriented according to orthogonal anatomical axes. Therefore, the approach to the study anatomical and functional of the heart provides an accurate orientation of the acquisition plans in relationship to the functional axes of the heart, also known from echocardiography.

\section{Anatomical Study of Large Vessels}

An important tool for vascular anatomical study is the three-dimensional angio-MR with contrast administration (CE-3D-MRA). Thanks to the high spatial resolution and wide field of view it displays with remarkable accuracy all mediastinal and thoracic vascular structures and is particularly suitable for it study of the complex anatomy of CCD. The bolus injection of $0.15-0.2 \mathrm{mmol} / \mathrm{kg}$ of a contrast based on gadolinium allows one quite fast regeneration of the longitudinal magnetization of the blood thus minimizing the saturation effect of repeated excitations. This allows a very fast acquisition of image data using ultra-fast sequences with repetition time (TR) and echo times (TE) as short as possible $(5 / 2 \mathrm{msec})$, as in 3D Spoiled Gradient Echo (SPGR) sequences. By using a relatively high flip angle between $30^{\circ}-60^{\circ}$ enough saturation of the is obtained. The temporal resolution is such as to be able to carry out the MRA also in spontaneous breath, adopted in small children in sedation. An important advantage of the technique is that the cardiac trigger is not necessarily required, but using the trigger allows us to get sharper images. With an adequate synchronization of the arrival of the contrast with the acquisition it is possible optimize and adapt the angiographic examination to the various vascular phases of interest (pulmonary circulation arterial, pulmonary venous, sinister cardiac sections with the aorta, abdominal venous return). In the adult we use a contrast concentration of $0.1 \mathrm{mmol} / \mathrm{kg}(20 \mathrm{ml})$ with a speed of injection of $2 \mathrm{ml} / \mathrm{sec}$, followed by $20 \mathrm{ml}$ of $\mathrm{NaCl}$. Angiographic images are subsequently processed depending on the clinical question in the form of MPR, MIP or surface reconstructions in special 3D processing programs after deleting parts of the image that are not of interest, such as for example. The skeletal structures of the thoracic cage with subcutaneous fat. Multiplanar reconstructions (MPR) are used for the measurement of vascular diameters and therefore for the quantitative evaluation of stenoses and aneurysms. The so-called "Whole Heart Coronary-MRA" allows the study of the coronary segments with variable visibility, better for the proximal segments [6].

\section{Study of the Biventricular Function}

The standardized method in the evaluation of the ventricular function - which allows a reference to normal values - it is the segmentation of the left ventricle proposed by the American Heart Association- used in echocardiography - modulated on the short axis of the heart, from the base to the apex [7,8]. 8-12 Cine-SSFP scans are acquired with a thickness of $6-10 \mathrm{~mm}$ with an interval of $0-4 \mathrm{~mm}$ (overall thickness $<1 \mathrm{~cm}$ ), oriented along the short cardiac axis covering the entire ventricle right and left from the atrioventricular plane to the apex. To obtain the ventricular volumes, they are plotted in a special segmentation software semiautomatic the endocardial contours in Cine-MR images acquired in short axis. The difference between end-diastolic volume and end systolic volume determines the stroke volume or "stroke volume" (SV) in ml, volume expelled in the systole [9]. The end-diastolic volume is a parameter of ventricular dilation, considered in the mild adult with values between $130-100 / \mathrm{m} 2$, moderate with values between $150-131 \mathrm{ml} / \mathrm{m} 2$ and severe with values $>150$ $\mathrm{ml} / \mathrm{m} 2$. The ejection fraction is considered the absolute parameter of ventricular contractility. We consider the systolic function conserved with an EF> 50\%, slightly depressed between 50-40\%, moderately depressed between $30-40 \%$ and severely depressed $<30 \%$. In the period between January 2016 and April 2018, they were studied with CMR 60 patients after surgical repair of CoA. 


\section{Clinical Radiology \& Imaging Journal}

\section{Study of Surgical Correction of Aortic Coarctation (AC)}

The most frequent surgical intervention in aortic coarctation is represented by the resection of the coarctated tract with termino-terminal anastomosis. This type of intervention is almost always possible and applicable, so that coarctation is not associated with an extended hypoplasia of the Aortic arch. Ductal tissue is also removed with the resection of the constricted tract. Another variant is the application of a prosthetic patch. This methodology finds employment in the child when the coarctated tract is present for a long stretch of aorta or in the newborn when the intervention is considered an emergency intervention. Other surgical technique is the subclavian flap with the use of the subclavian artery as a flap for the reconstruction of the aorta in his stretched trait. This technique involves first the sacrifice of the upper limb artery left and then needs a subclavian with adequate course size. In patients under the age of one the sacrifice of the subclavian artery does not bring any details problems as the collateral circle that develops following the binding, ensures one enough nutrition of the left upper limb. The prosthetic tube technique is used to replace the constricted aortic tract and is a technique today almost abandoned in the pediatric age, which is still taken into consideration in the patient adult. Cardiology can be considered in the treatment of aortic coarctation interventional with the possibility of positioning one or more stents.

The study of surgical correction of AC aims at the exclusion of complications, such as the re-establishment, residual stenosis, pseudoaneurysm of the patch or residual hypoplasia of the aortic arch. In some cases, it is also necessary to evaluate the left ventricular function and if aortic valve abnormalities are associated, such as the bicuspid aorta. Other postoperative complications are represented by wall complications aortic itself ("aortic wall complications"), like true and false aneurysms, the dissection of aortic wall, endarteritis or fistulas. To identify these problems, we use Cine -True-fisp (Phase Contrast) for the flow-study and the Angio-RM 3D for the morphological study. The Phase-Contrast technique is then used to calculate the gradient at the level of the stenosis maximum, obtaining a flow velocity value in $\mathrm{cm}$ / sec, using the Bernoulli formula (DP $=4 \mathrm{~V} 2$ ). With the value of the gradient at the level of the stenosis the possibility of can be evaluated a surgical or interventional intervention. These techniques are used both in the study preoperative than in the evaluation of postoperative complications. The Angio - RM 3D is used to measure the thoracic aorta diameters. When a significant stenosis is present, a stenosis $>50 \%$, calculating the normal lumen at level of the diaphragmatic aorta, a reintervention at this level is indicated [10]. According to other authors we must already consider a significant stenosis a value $>40 \%$ [11]. Other authors speak of a significant stenosis, using the ratio of the diameter of the stenosis with respect to the diameter of the supradiaphragmatic aorta. A value $<0.7$ indicates a stricture to be reprocessed [12]. The analysis of the flow profile, where yes, will also help the assessment of restenosis will speak of a significant stenosis with a peak velocity greater than $3 \mathrm{~m} / \mathrm{sec}-"$ peak velocity" 3m / sec + diastolic tail" [11]. The identification of an aneurysm at thoracic aorta is also important. In this case not it counts both the diameter of the aortic wall dilation, but above all the relationship between diameter of the aneurysm and diameter at the level of the supradiaphragmatic aorta. If there is a value of $>1.5$ we will speak of an aneurysmal dilatation (Table 1, Figures 18).

\begin{tabular}{|c|c|c|c|c|}
\hline & TT (n= 37) & Patch (n= 13) & $\begin{array}{c}\text { Subclavian Flap } \\
(\mathbf{n = 3})\end{array}$ & $\begin{array}{c}\text { Prosthetic tube graft } \\
(\mathbf{n}=\mathbf{6})\end{array}$ \\
\hline Median age & $24(1-52)$ & $33(13-58)$ & $21(16-27)$ & $33,5(23-50)$ \\
\hline Residual Stenosis & 7 & 7 & 0 & 0 \\
\hline Restenosis & 8 & 1 & 0 & 0 \\
\hline Hypoplastic aortic arch & 9 & 1 & 1 & 0 \\
\hline Dilatation/ Aneurysm & $0 / 1$ & $8 / 1$ & $0 / 0$ & $0 / 0$ \\
\hline Dissection/ thrombosis & 0 & $0 / 1$ & $0 / 0$ & $0 / 0$ \\
\hline LV dilatation & 4 & 2 & 2 & 1 \\
\hline AI (ARF \%) & 13 & 7 & 1 & 2 \\
\hline
\end{tabular}


Clinical Radiology \& Imaging Journal

\begin{tabular}{|c|c|c|c|c|}
\hline Reintervention & $\begin{array}{c}\text { - substitution aortic } \\
\text { valve }(n=4) \\
\text { - reintervention for } \\
\text { recoarctation }(n=1) \\
\text {-percutan } \\
\text { valvulotomy }(n=1) \\
\text {-prothesic tube } \\
\text { implantation }(n=1)\end{array}$ & $\begin{array}{l}\text {-implantation of vascular prosthesis } \\
\text { aortic arch }(\mathrm{n}=1) \\
\text {-percutan implantation of stent in } \\
\text { descending aorta for } \\
\text { dissection(n=1) }\end{array}$ & 0 & 0 \\
\hline PTA & 7 & 1 & 1 & 0 \\
\hline
\end{tabular}

Table 1: Follow Up of Surgical Correction of Aortic Coarctation $(n=60)$.

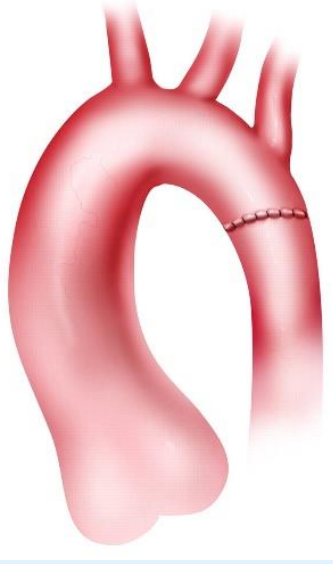

Figure 1: Illustration shows end-to-end anastomosis for repair of coarctation of aorta.



Figure 2: Illustration shows patch plastic for repair of coarctation of aorta with incision site for patch repair (dashed line).
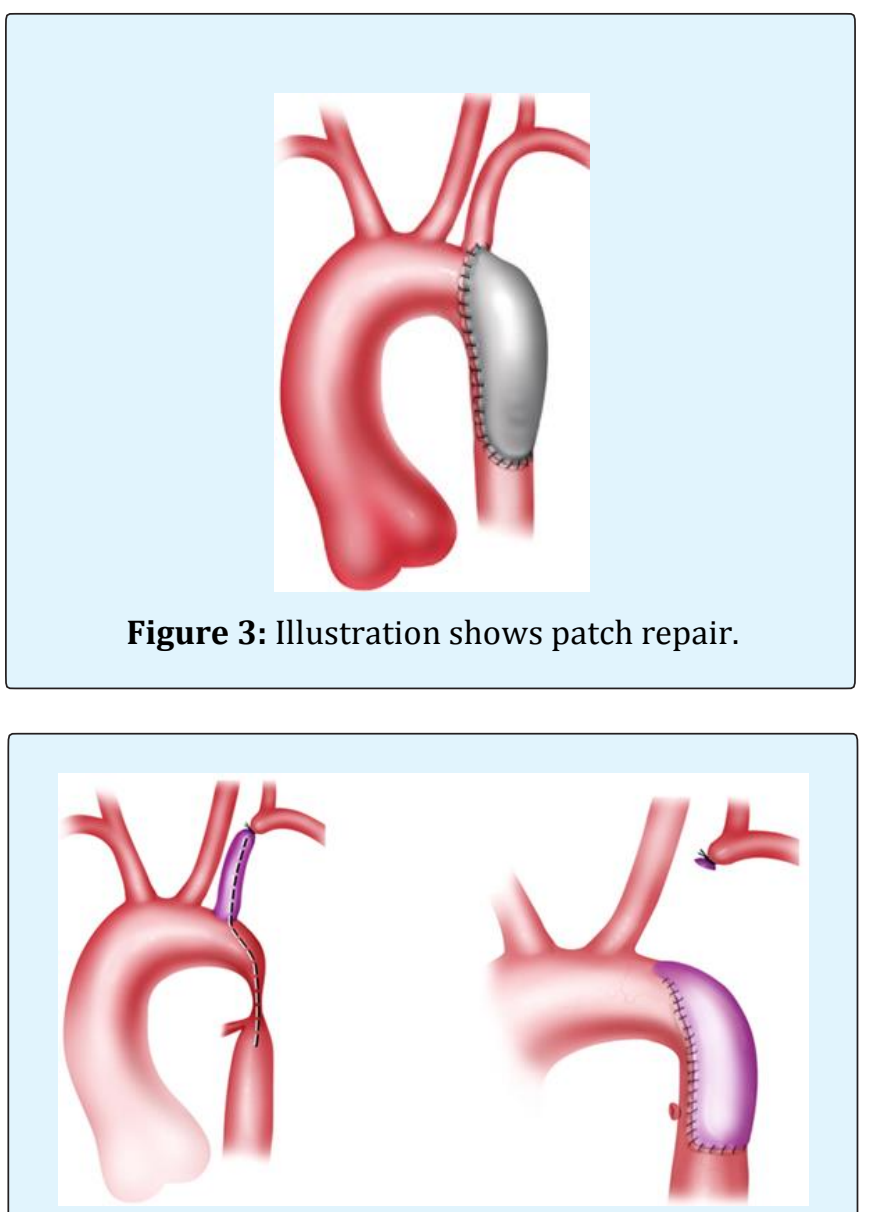

Figure 4

Figure 5

Figures 4 \& 5: Illustrations show Waldhausen repair of aortic coarctation. 


\section{Clinical Radiology \& Imaging Journal}
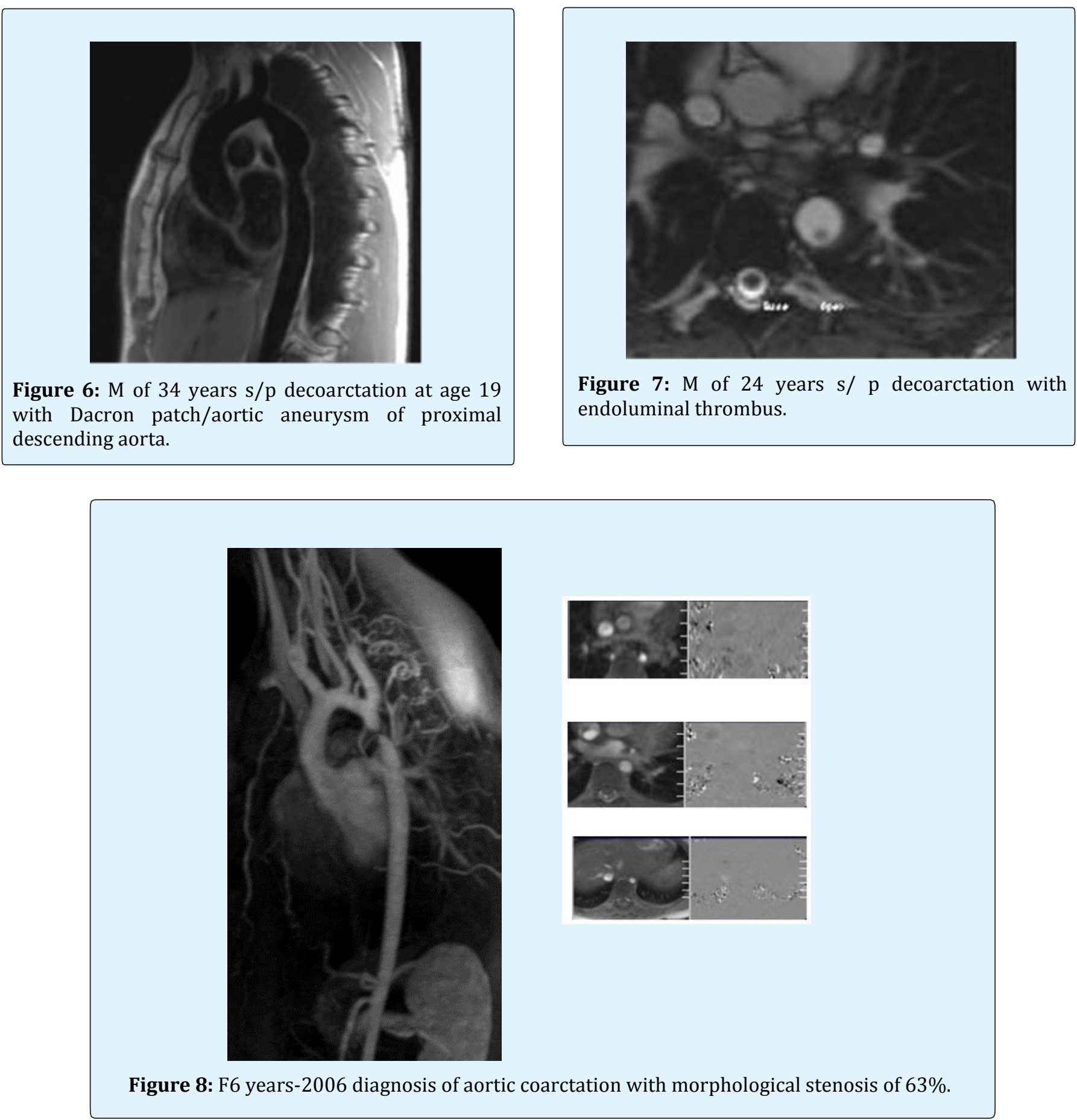

\section{Conclusion}

Over the last years a new scenario for imaging diagnostics has opened up in the approach to the postsurgical study of aortic coarctation. If in the past the role of imaging was predominantly based on the identification of cardiac malformations and consequently on the morphological data, today prognostic evaluation and follow-up have become fundamental elements, particularly in the adults, requiring a greater wealth of information, even functional and a method of 


\section{Clinical Radiology \& Imaging Journal}

investigation as accurate, reproducible and above all noninvasive as possible. These features, and the noninvasiveness and the high reproducibility of the parameters, make the CRM an ideal method for the morphological and functional study of congenital cardiac diseases, considering the progressive evolution of the characteristics of the study population.

\section{References}

1. Hoffmann JI (1995) Incidence of congenital heart disease: I. postnatal incidence. Pediatr Cardiol (1995) 16(3): 103-113.

2. American Heart Association (2004) Heart Disease and stroke Statistics, Dallas, Texas.

3. Pruessmann KP, Geiger M, Scheidegger MB, Boesiger $P$ (1999) SENSE: sensitività encoding for fast MRI. Magn Reson Med 42(5): 952-962.

4. Finn JP, Neal K, Deshpande V, Ratib O, Laub G (2006) Cardiac MR Imaging: state of technology. Radiology 241(2): 338-354.

5. Chavhan GB, Babyn SP, Jankharia BG, Cheng HLM, Shroff MM (2008) Steady State MR Imaging Sequences: Physics, Classification, and Clinical Applications. Radio Graphics 28(4): 1147-1160.

6. So NM, Lam W, Li D, Chan AK, Sanderson JE, et al. (2005) Magnetic resonance angiography of coronary arteries with a 3-dimensional magnetizationprepared true fast imaging with steady-state precession sequenze compared with conventional coronary angiography. Am Heart J 150(3): 530-535.

7. Alfakih K, Reid S, Jones T, Sivanathan M (2004) Assessment of ventricular function and mass by cardiac magnetic resonance imaging. Eur Radiol 14(10): 1813-1822.

8. Cerqueira MD, Weissman NJ, Dilsizian V, Jacobs AK, Kaul S, et al. (2002) Standardized Myocardial Segmentation and Nomenclature for Tomographic Imaging of the Heart: A Statement of Healthcare Professionals from the Cardiac Imaging Committee of the Council on Clinical Cardiology of the American Heart Association. Circulation 105(4): 539-542.

9. Higgins CB, De Roos A (2002) Cardiovascolar MRI and MRA. Lippincott Williams \& Wilkins.

10. Eicken A, Pensl U, Sebening W, Hager A, Genz T (2006) The fate of systemic blood pressure in patients after effectively stented coarctation. Eur Heart J 27(9): 1100-1105.

11. Eichhorn JG, Fink C, Delorme S, Hagl S, Kauczor HU, et al. (2006) Magnetic resonance blood flow measurements in the follow-up of pediatric patients with aortic coarctation-a re-evaluation. Int J Cardiol 113(3): 291-298.

12. Vriend JW, Zwinderman AH, de Groot E, Kastelein JJ, Bouma BJ, et al. (2005) Predictive value of mild, residual descending aortic narrowing for blood pressure and vascular damage in patients after repair of aortic coarctation. Eur Heart J 26(1): 84-90. 